\title{
Convergence Analysis of a Background Interstage Gain Calibration Technique for Pipelined ADCs*
}

\author{
Dong Wang, John P. Keane, Paul J. Hurst, Bernard C. Levy and Stephen H. Lewis \\ Department of Electrical and Computer Engineering \\ University of California, Davis, CA 95616
}

\begin{abstract}
A mathematical framework for the convergence analysis of a pipelined ADC with background gain calibration is presented. The constraints on adaptation step size for mean convergence, for meansquared convergence, and for signal-to-adaptation-noise ratio are derived. Furthermore, expressions for steady-state tap noise and for signal-to-adaptation-noise ratio are derived. The analysis results are verified with simulations.
\end{abstract}

\section{INTRODUCTION}

An analog-to-digital converter (ADC) compares an input sample $V_{i n}$ at time $n$ to a reference voltage $V_{\text {ref }}$ to generate a digital approximation $i_{d}[n]$ of the normalized input $i[n]=V_{i n}[n] / V_{\text {ref }}$. A pipelined ADC is shown in Fig. 1. All signals are normalized to $V_{r e f}$, so the input range is $(-1,+1)$. The analog-to-digital subconverter (ADSC) generates an $M$-level digital estimation $D[n]$ of $i[n]$ by comparing it to a set of $M-1$ threshold levels. Using a digital-to-analog sub-converter (DASC), this digital word is then converted to an analog signal that is subtracted from $i[n]$ to form the residue $z[n]$. The residue is the input to a back-end $\mathrm{ADC}\left(\mathrm{ADC}_{\mathrm{BE}}\right)$, which generates a digital output $z_{d}[n]$ that is a digital estimate of the back-end ADC input. The back-end ADC consists of an interstage amplifier with gain $1 / m_{0}$ that generates an output $y[n]$, which is then quantized to produce $y_{d}[n]$. Assuming an ideal DASC with gain of one, the ADC output is calculated by

$$
i_{d}[n]=D[n]+m_{0} y_{d}[n] .
$$

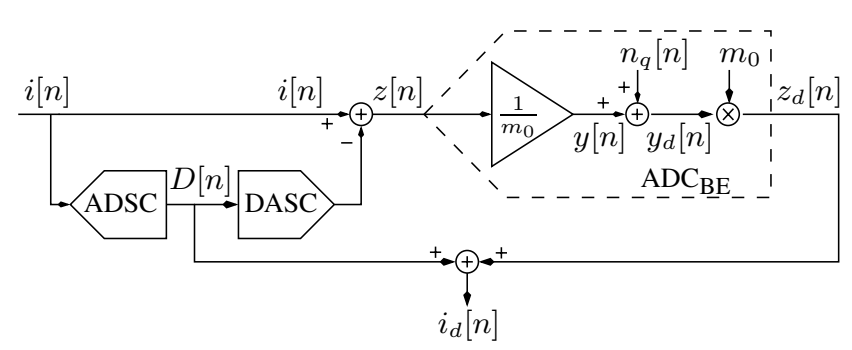

Fig. 1. Traditional pipelined ADC.

To prevent $z[n]$ from exceeding the input range of $\mathrm{ADC}_{\mathrm{BE}}$, the magnitude of the residue $z[n]$ must be less than $m_{0}$. Even with an optimal spacing of ADSC and DASC levels, this requires that $M \geq 1 / m_{0}$. In practice, due to errors in the stage ADSC and offsets present in the stage, over-range is possible, so a value of $M>1 / m_{0}$ is often used. This choice allows large comparator offsets without affecting converter linearity and is referred to as redundancy with digital correction [1]. When this redundancy is

\footnotetext{
*This research was supported by UC MICRO Grant No. 03-051.
}

present, the linearity of the ADC transfer function can be independent of large errors in the ADSC thresholds. The primary remaining sources of linearity error are errors in the interstage amplifier and nonlinearity in the DASC. In a switched-capacitor implementation, DASC nonlinearity can be measured and corrected in the digital domain [2]. Hence, the primary source of ADC nonlinearity is due to errors in the interstage gain $1 / m_{0}$. These errors stem from capacitor mismatch, insufficient op amp gain and charge injection. To address this problem, digital self-calibration is often used, where the value of $m_{0}$ in (1) is adjusted to match the actual interstage gain.

A background calibration technique to correct for interstage gain errors was introduced in [3] and extended in [4], [5]. In this method, a randomly modulated calibration signal is added to the DASC output in a pipelined stage, and its effect is measured by correlating the ADC output with the same modulation sequence. If the amplitude of the calibration signal is known, then the magnitude of $m_{0}$ can be estimated.

\section{Gain CALibRATED ADC}

The background gain calibration technique presented in [3], [5] is summarized by Fig. 2. A dither $R[n] \Delta D$ is added to both the output of the DASC and to the digital code $D[n]$, where $R[n]$ is a random sequence of $\pm 1, n$ is a time index, and $\Delta D$ is a constant. This dither can be added using a digital-to-analog converter $\left(\mathrm{DAC}_{\mathrm{d}}\right)$. In [4], [5], the dither is added in the digital domain to the ADSC output $D[n]$, so that the DASC and the $\mathrm{DAC}_{\mathrm{d}}$ are combined. The dither thus introduced is used for gain calibration.

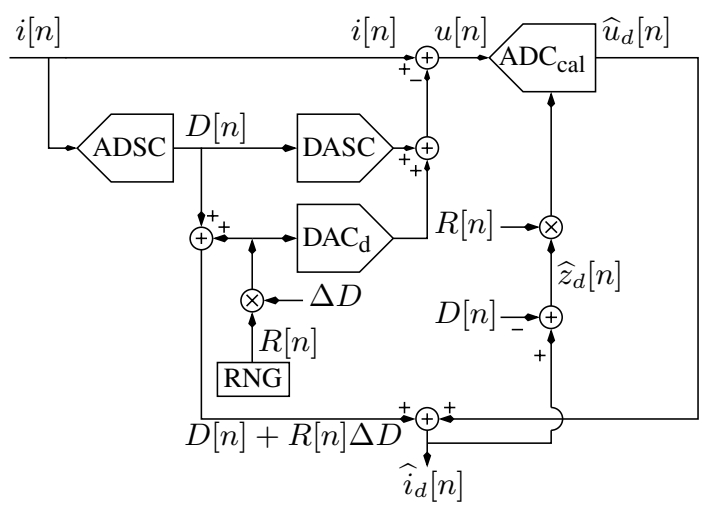

Fig. 2. ADC with gain calibration; $\mathrm{ADC}_{\mathrm{cal}}$ is defined in Fig. 4.

Fig. 2 is difficult to analyze since the transfer function from $i[n]$ to $u[n]$ is nonlinear. To simplify the analysis, Fig. 2 is transformed as shown in Fig. 3. The advantage of the system in Fig. 3 is that it consists of a traditional nonlinear part followed by a linear $\mathrm{ADC}_{\mathrm{BE}}$. The nonlinear part is not involved in calibration. Now the calibrating $\mathrm{ADC}\left(\mathrm{ADC}_{\mathrm{cal}}\right)$ and the added dither are contained within $\mathrm{ADC}_{\mathrm{BE}}$. 


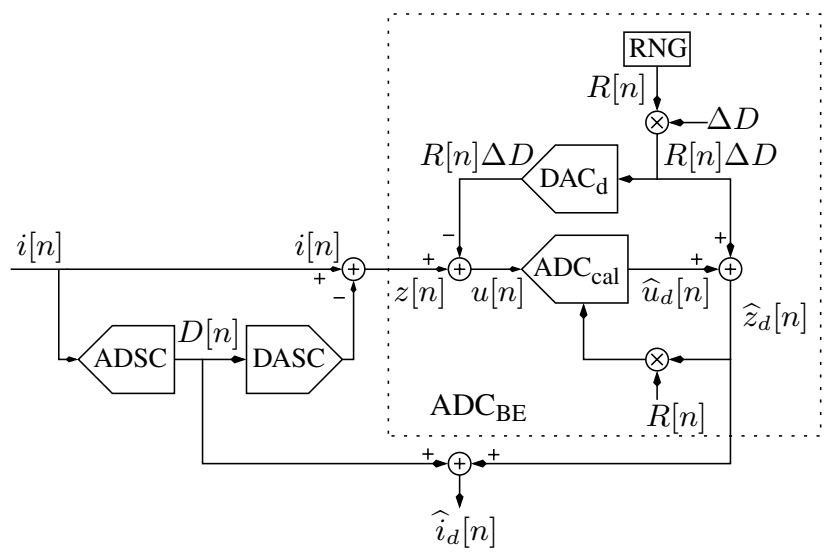

Fig. 3. $\mathrm{ADC}$ separated into linear $\mathrm{ADC}_{\mathrm{BE}}$ and nonlinear parts.

The analysis in this paper will focus on a linear model of $\mathrm{ADC}_{\mathrm{BE}}$ as shown in Fig. 4. Some definitions are given in Table I. When $\widehat{m}[n-1]=m_{0}, z_{d}[n]$ is defined as $\widehat{z}_{d}[n]$ in Fig. 4, and

$$
z_{d}[n] \triangleq y_{d}[n] m_{0}+R[n] \Delta D
$$

with

$$
y_{d}[n]=\frac{z[n]}{m_{0}}-\frac{R[n] \Delta D}{m_{0}}+n_{q}[n] .
$$

Substituting (3) into (2) gives:

$$
z_{d}[n]=z[n]+m_{0} n_{q}[n] .
$$

The estimated quantized-residual error $\left(\widetilde{z}_{d}[n]\right)$ is defined as

$$
\widetilde{z}_{d}[n] \triangleq z_{d}[n]-\widehat{z}_{d}[n] \text {. }
$$

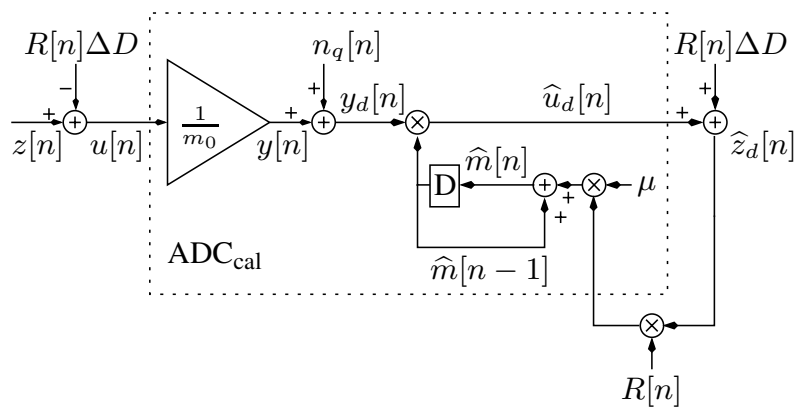

Fig. 4. Model of $\mathrm{ADC}_{\mathrm{BE}}$ including $\mathrm{ADC}_{\mathrm{cal}}$.

TABLE I

DEFINITION OF TERMS.

\begin{tabular}{l|l}
\hline Term & Notation \\
\hline 1/(Amplifier gain) & $m_{0}$ \\
Estimate of $m$ & $\widehat{m}[n]$ \\
Error in estimate of $m$ & $\widetilde{m}[n]$ \\
\hline Residual & $z[n]$ \\
Quantized residual & $z_{d}[n]$ \\
Estimate of quantized residual & $\widehat{z}_{d}[n]$ \\
Error in estimate of quantized residual & $\widetilde{z}_{d}[n]$ \\
\hline Residual for calibration & $u[n]$ \\
Estimate of quantized residual for calibration & $\widehat{u}_{d}[n]$ \\
\hline Dither amplitude & $\Delta D$ \\
Random sequence of $\{+1,-1\}$ & $R[n]$
\end{tabular}

This analysis assumes the binary sequence $R[n]$ is white and stationary with zero mean. The residual sequence $z[n]$ is also assumed to be white and stationary. In addition, $z[j]$ is assumed to be independent of $R[k]$ for all possible combinations of $j, k$.
The analysis is divided into three steps. Section 3 starts with the mean convergence analysis. Mean-squared convergence is analyzed in Section 4. Finally, steady-state performance is analyzed in Section 5 .

\section{MEAN CONVERGENCE}

A constraint on adaptation step-size $(\mu)$ for mean convergence is derived in this section. Start with the update equation as defined in [5] and shown in Fig. 4:

$$
\widehat{m}[n] \triangleq \widehat{m}[n-1]+\mu R[n] \widehat{z}_{d}[n] .
$$

Subtracting $m_{0}$ from both sides and noting that $\widetilde{m}[n] \triangleq m_{0}-\widehat{m}[n]$ gives:

$$
\widetilde{m}[n]=\widetilde{m}[n-1]-\mu R[n] \widehat{z}_{d}[n] .
$$

Substituting $\widehat{z}_{d}[n]$ from (5) into (7) gives:

$$
\widetilde{m}[n]=\widetilde{m}[n-1]-\mu R[n]\left(z_{d}[n]-\widetilde{z}_{d}[n]\right) .
$$

From Fig. 4 and (5), $\widetilde{z}_{d}[n]$ can be shown to be:

$$
\widetilde{z}_{d}[n]=\widetilde{m}[n-1] y_{d}[n] .
$$

Substituting $\widetilde{z}_{d}[n]$ from (9) into (8) and simplifying results in:

$$
\widetilde{m}[n]=\widetilde{m}[n-1] A[n]-\mu R[n] z_{d}[n],
$$

where

$$
A[n] \triangleq 1+\mu R[n] y_{d}[n] .
$$

Taking the expectation of (10) and noting that $A[n]$ is independent of $\widetilde{m}[n-1]$ gives:

$$
E\{\widetilde{m}[n]\}=E\{\widetilde{m}[n-1]\} E\{A[n]\}-\mu E\left\{R[n] z_{d}[n]\right\} .
$$

Expanding (12) using (11), (3), and (4), then further noting that $R[n]$ is independent of $z[n]$ and has zero mean, results in:

$$
\begin{aligned}
E\{\tilde{m}[n]\}= & E\{\tilde{m}[n-1]\}\left(1-\mu \frac{\Delta D}{m_{0}}\right) \\
& +\mu E\left\{R[n] n_{q}[n]\right\}\left(E\{\widetilde{m}[n-1]\}-m_{0}\right) .
\end{aligned}
$$

It is generally true [5] that if the input signal varies enough to provide sufficient dither, then

$$
E\left\{R[n] n_{q}[n]\right\} \approx 0 .
$$

When (14) is true, (13) reduces to:

$$
E\{\widetilde{m}[n]\}=E\{\widetilde{m}[n-1]\} B,
$$

where

$$
B \triangleq 1-\mu \frac{\Delta D}{m_{0}} .
$$

Eq. (15) can be re-written in closed form as:

$$
E\{\widetilde{m}[n]\}=E\{\widetilde{m}[0]\} B^{n} .
$$

From (17), it can be seen that the sequence $E\{\widetilde{m}[n]\}$ converges if $-1<B<1$. This constrains $\mu$ to be in the range

$$
\mathbf{0}<\mu<\mathbf{2} \mathbf{m}_{\mathbf{0}} / \Delta \mathrm{D} \text {. }
$$

Thus when (18) is satisfied, $E\{\widehat{m}[n]\}$ converges to $m_{0}$.

It is interesting to note that $E\{\widehat{m}[n]\}$ can converge to $m_{0}$ in one time step when $\mu=\mu_{\text {opt }} \triangleq m_{0} / \Delta D[6$, p. 180]. In practice, the optimum $\mu\left(\mu_{\text {opt }}\right)$ cannot be used due to other limitations. A large $\mu$ can cause excessive error power in $\widehat{m}[n]\left(E\left\{\widetilde{m}^{2}[n]\right\}\right)$ and excessive noise power in $\widehat{z}_{d}[n]\left(E\left\{\widetilde{z}_{d}^{2}[n]\right\}\right)$. In some cases, it can cause $E\left\{\widetilde{m}^{2}[n]\right\}$ and $E\left\{\widetilde{z}_{d}^{2}[n]\right\}$ to diverge as shown in the next two sections. 


\section{MEAN-Squared CONVERGENCE}

The mean convergence criterion as shown in (18) is a necessary but not a sufficient condition for the safe operation of the adaptive calibration in (6). The criterion for mean-squared convergence $\left(\lim _{n \rightarrow \infty} E\left\{\widetilde{m}^{2}[n]\right\}<\infty\right)$ is also relevant in addition to the criterion for mean convergence $\left(\lim _{n \rightarrow \infty} E\{\widetilde{m}[n]\} \rightarrow 0\right)$.

The criterion for mean-squared convergence is derived below. The steps in the derivation are: finding a relation between $\widetilde{m}^{2}[n]$ and $\widetilde{m}^{2}[n-1]$, taking expectation of $\widetilde{m}^{2}[n]$, relating $E\left\{\widetilde{m}^{2}[n]\right\}$ to $E\left\{\widetilde{m}^{2}[n-1]\right\}$, deriving an expression for $E\left\{\widetilde{m}^{2}[n]\right\}$ assuming mean convergence, and finding the convergence criterion for the sequence $E\left\{\widetilde{m}^{2}[n]\right\}$.

To find the relation between $\widetilde{m}^{2}[n]$ and $\widetilde{m}^{2}[n-1]$, start by squaring both sides of (10) to get:

$$
\begin{aligned}
\widetilde{m}^{2}[n]=\widetilde{m}^{2}[n-1] A^{2}[n] & +\mu^{2} z_{d}^{2}[n] \\
& -2 \mu R[n] \widetilde{m}[n-1] A[n] z_{d}[n] .
\end{aligned}
$$

Taking the expectation of (19) and noting that $\tilde{m}[n-1]$ is independent of $A[n]$, and $\widetilde{m}[n-1]$ is independent of $R[n] A[n] z_{d}[n]$ gives:

$$
\begin{array}{r}
E\left\{\widetilde{m}^{2}[n]\right\}=E\left\{\widetilde{m}^{2}[n-1]\right\} E\left\{A^{2}[n]\right\}+\mu^{2} E\left\{z_{d}^{2}[n]\right\} \\
-2 \mu E\{\widetilde{m}[n-1]\} E\left\{R[n] A[n] z_{d}[n]\right\} .
\end{array}
$$

Assuming mean convergence,

$$
E\left\{\widetilde{m}^{2}[n]\right\} \stackrel{\text { mean conv. }}{\longrightarrow} E\left\{\widetilde{m}^{2}[n-1]\right\} C+D,
$$

where

$$
C \triangleq E\left\{A^{2}[n]\right\}
$$

and

$$
D \triangleq \mu^{2} E\left\{z_{d}^{2}[n]\right\} .
$$

The expression in (21) can be re-written as:

$$
E\left\{\widetilde{m}^{2}[n]\right\} \stackrel{\text { mean conv. }}{\longrightarrow} E\left\{\widetilde{m}^{2}[0]\right\} C^{n}+D \sum_{j=0}^{j=n-1} C^{j}
$$

From (24), it can be seen that the sequence $E\left\{\widetilde{m}^{2}[n]\right\}$ converges if

$$
-1<C<1 \text {. }
$$

Next, an expanded expression for $C$ is derived to find the constraint on $\mu$ for mean-squared convergence. Start by squaring $A[n]$ as defined in (11) to get

$$
A^{2}[n]=1+2 \mu R[n] y_{d}[n]+\mu^{2} y_{d}^{2}[n] .
$$

Expanding the right hand side of (26) using (3), taking expectation, and using (14) gives:

$$
C \triangleq E\left\{A^{2}[n]\right\}=1-2 \mu \frac{\Delta D}{m_{0}}+\mu^{2} E\left\{y_{d}^{2}[n]\right\} .
$$

With (25) and (27), it can be shown that the constraint on $\mu$ for mean-squared convergence is:

$$
\mu<\frac{\mathbf{2} \Delta \mathbf{D} / \mathbf{m}_{\mathbf{0}}}{\mathbf{E}\left\{\mathbf{y}_{\mathrm{d}}^{2}[\mathbf{n}]\right\}}
$$

When this inequality is satisfied, the mean-squared error in $\widehat{m}[n]$ does not diverge. This result does not imply that $E\left\{\widetilde{m}^{2}[n]\right\}$ converges to zero. In Section 5, the steady-state value for $E\left\{\widetilde{m}^{2}[n]\right\}$ is found.

\section{STEADY-STATE PERFORMANCE}

In the previous two sections, expressions were derived for mean and mean-squared convergence. In Section 5-A an expression for steady-state adaptation noise in $\widehat{m}[n]$ is derived assuming meansquared convergence. Furthermore, steady-state signal-to-adaptionnoise ratio $\left(\mathrm{SNR}_{\mathrm{SS}}\right)$ and its limitation on $\mu$ are derived in Section 5-B.

\section{A. Mean-squared error}

Assuming mean-squared convergence, steady-state mean-squared error can be found by taking the limit of (24) as time $n$ tends to infinity to give:

$$
\lim _{n \rightarrow \infty} E\left\{\widetilde{m}^{2}[n]\right\}=\frac{D}{1-C} .
$$

Plugging $C$ from (27) and $D$ from (23) into (29) gives the steadystate mean-squared error in $\widehat{m}[n]$ :

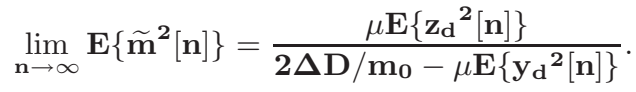

For small $\mu$, (30) can be approximated by

$$
\lim _{n \rightarrow \infty} E\left\{\widetilde{m}^{2}[n]\right\}=\frac{\mu E\left\{z_{d}^{2}[n]\right\}}{2 \Delta D / m_{0}},
$$

and $\widetilde{m}[n]$ has a Gaussian distribution [7, pp. 103-107].

Section 5-B will show that $\mu$ is limited by the SNR requirement on $\mathrm{ADC}_{\mathrm{BE}}$. In practice this is often the most severe limitation on $\mu$.

\section{B. signal-to-adaptation-noise ratio}

The steady-state signal-to-adaptation-noise ratio at the output of $\mathrm{ADC}_{\mathrm{BE}}$ is derived below. First, steady-state signal power is defined as:

$$
Q_{S_{s s}} \triangleq \lim _{n \rightarrow \infty} E\left\{z_{d}^{2}[n]\right\} .
$$

Next, adaptation noise is defined here as $\widetilde{z}_{d}[n]$. Finally, steady-state adaptation noise power is defined as:

$$
Q_{N_{s s}} \triangleq \lim _{n \rightarrow \infty} E\left\{\widetilde{z}_{d}^{2}[n]\right\} .
$$

Plugging $\widetilde{z}_{d}[n]$ from (9) into (33) and noting that $\widetilde{m}[n-1]$ is independent of $y_{d}[n]$ gives:

$$
Q_{N_{s s}}=\left(\lim _{n \rightarrow \infty} E\left\{\widetilde{m}^{2}[n-1]\right\}\right) E\left\{y_{d}^{2}[n]\right\} .
$$

Plugging (30) into (34) gives:

$$
Q_{N_{s s}}=\left(\frac{\mu E\left\{z_{d}^{2}[n-1]\right\}}{2 \Delta D / m_{0}-\mu E\left\{y_{d}^{2}[n-1]\right\}}\right) E\left\{y_{d}^{2}[n]\right\} \text {. }
$$

Steady-state signal-to-adaptation-noise ratio is defined as:

$$
S N R_{s s} \triangleq \frac{Q_{S_{s s}}}{Q_{N_{s s}}} .
$$

Substituting (32) for $Q_{S_{s s}}$ and (35) for $Q_{N_{s s}}$ into (36) gives:

$$
S N R_{s s}=\frac{E\left\{z_{d}^{2}[n]\right\}}{E\left\{z_{d}^{2}[n-1]\right\}} \frac{2 \Delta D / m_{0}-\mu E\left\{y_{d}^{2}[n-1]\right\}}{\mu E\left\{y_{d}^{2}[n]\right\}} .
$$

Noting that both $z_{d}^{2}[n]$ and $y_{d}^{2}[n]$ are stationary gives:

$$
S N R_{s s}=\frac{2 \Delta D / m_{0}-\mu E\left\{y_{d}^{2}[n]\right\}}{\mu E\left\{y_{d}^{2}[n]\right\}} .
$$

Eq. (38) further simplifies to:

$$
\mathbf{S N R}_{\mathrm{ss}}=\frac{2}{\mu} \frac{\Delta \mathrm{D} / \mathbf{m}_{0}}{\mathbf{E}\left\{\mathbf{y}_{\mathrm{d}}^{2}[\mathbf{n}]\right\}}-1 .
$$

Eq. (39) shows that $\mathrm{SNR}_{\mathrm{SS}}$ is inversely proportional to $\mu$. Thus, constraining $\mathrm{SNR}_{\mathrm{SS}}$ by a lower bound $\left(\mathrm{SNR}_{\min }\right)$ would imply an 
upper bound for $\mu\left(\mu_{\max }\right)$. This can be shown by re-arranging (39) to get:

$$
\mu<\mu_{\max }=2 \frac{\Delta \mathbf{D} / \mathbf{m}_{\mathbf{0}}}{\mathbf{E}\left\{\mathbf{y}_{\mathbf{d}}^{2}[\mathbf{n}]\right\}} \frac{1}{\mathbf{S N R}_{\min }+\mathbf{1}} .
$$

Eq. (40) allows the designer to pick $\mu$ and $\Delta D$ to satisfy a specific $\mathrm{SNR}$ requirement for $\mathrm{ADC}_{\mathrm{BE}}$. In the next section, a practical example is simulated to verify the analysis.

\section{Simulation Results}

A back-end $\mathrm{ADC}\left(\mathrm{ADC}_{\mathrm{BE}}\right)$ with parameters listed in Table II was simulated. $R[n]$ is a sequence of independent and identically distributed (iid) random \pm 1 s with zero mean $(E\{R[n]\}=0) . z[n]$ is a sequence of iid random real numbers uniformly distributed within the interval $\left[\frac{-1}{16}, \frac{1}{16}\right)$. Furthermore, $z[j]$ is independent of $R[k]$ for all possible combinations of $j, k$. The constraints on $\mu$ for this particular setup are calculated to be: $\mu<4$ for mean convergence, $\mu<3$ for mean-squared convergence, and $\mu<1.1 \times 10^{-5}$ for 9 -bit SNR.

TABLE II

PARAMETERS For SIMULATED ADC $\mathrm{BE}$

\begin{tabular}{l|l||l|l}
\hline Parameter & Value & Parameter & Value \\
\hline Stages & 9 & bits & 9 \\
Slicers per Stage & 2 & $\Delta D$ & $\frac{1}{16}$ \\
$1 / m_{0}$ for first stage & 8 & $1 / m_{0}$ for last 8 stages & 2 \\
Range for $z[n]$ & {$\left[\frac{-1}{16}, \frac{1}{16}\right)$} & Range for $y[n]$ & {$[-1,1)$}
\end{tabular}
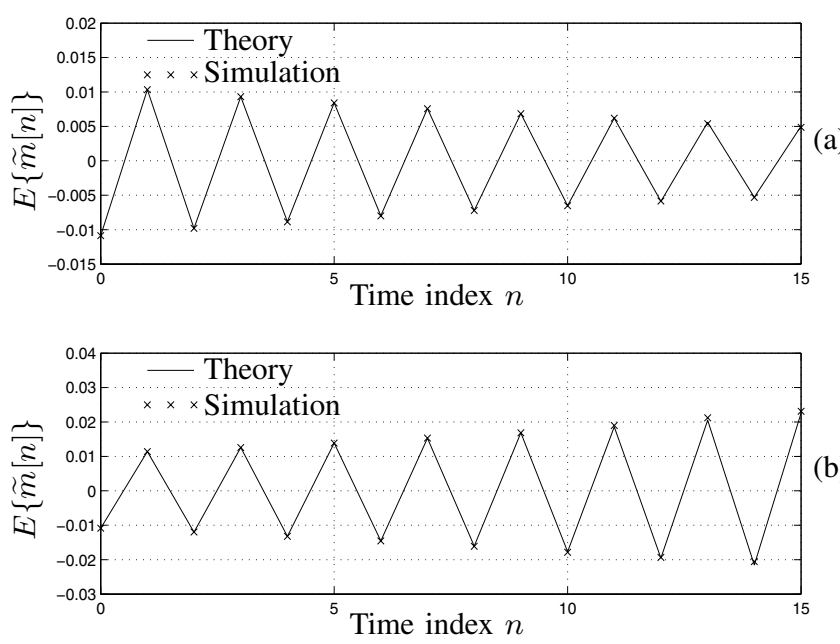

Fig. 5. $E\{\widehat{m}[n]\}$ versus time index for a) $\mu=3.9$, b) $\mu=4.1$.

Fig. 5 shows the theoretical and simulated expected value of the error in $\widehat{m}[n]$ for two cases: with $\mu=3.9$ (where it converges) and with $\mu=4.1$ (where it diverges). Fig. 6 shows the theoretical and simulated expected value of the mean-squared error in $\widehat{m}[n]$ for two cases: with $\mu=2.9$ (where convergence is reached) and with $\mu=3.1$ (where divergence occurs). Both Fig. 5 and Fig. 6 show good agreement between theory and simulation.

Fig. 7 plots the theoretical and simulated signal-to-adaptationnoise ratio (SNR) for $\mathrm{ADC}_{\mathrm{BE}}$ with $\mu=10^{-6}$. Furthermore, SNR including both quantization noise and adaptation noise $\left(\mathrm{SNR}_{\text {total }}\right)$ is also plotted. This plot shows that when adaptation noise is high, $\mathrm{SNR}_{\text {total }}$ is dominated by adaptation noise; when adaptation noise is low, $\mathrm{SNR}_{\text {total }}$ is dominated by quantization noise.
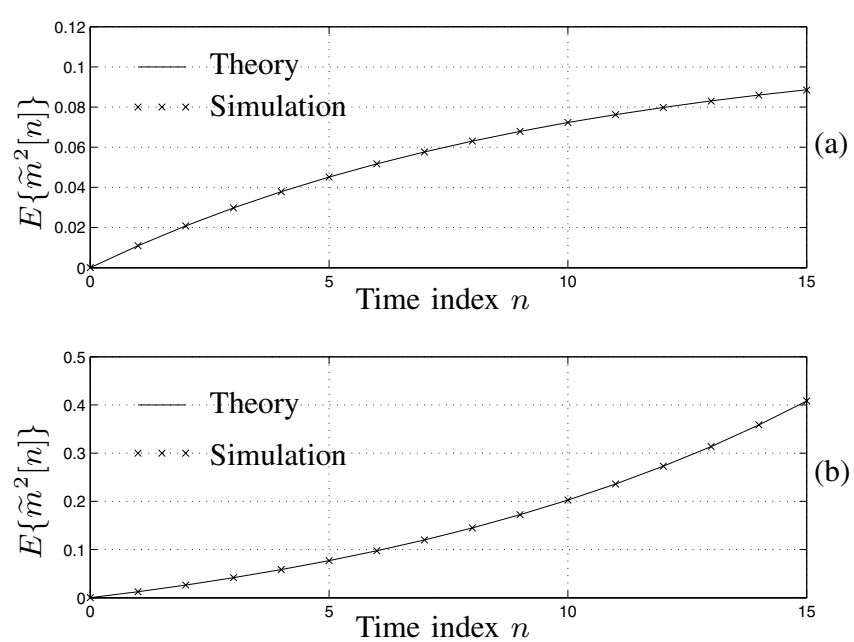

Fig. 6. Tap-noise power versus time index for a) $\mu=2.9$, b) $\mu=3.1$.

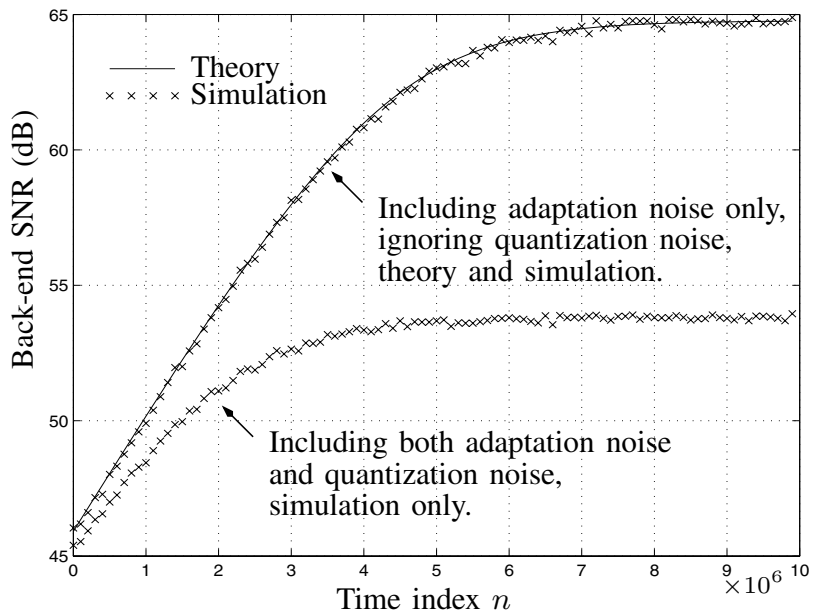

Fig. 7. Back-end SNR versus time index for $\mu=10^{-6}$.

\section{Conclusion}

The gain-calibrated ADC as described in [5] was analyzed. Three constraints on $\mu$ were derived: a mean convergence constraint, a mean-squared convergence constraint, and a constraint due to SNR limitations. All three were verified with simulations. For most practical applications, $\mu$ is constrained by SNR as shown in (40).

\section{REFERENCES}

[1] S. H. Lewis and P. R. Gray, "A pipelined 5-Msample/s 9-bit analogto-digital converter," IEEE J. of Solid-State Circuits, pp. 954-961, Dec. 1987

[2] I. Galton, "Digital cancellation of D/A converter noise in pipelined A/D converters," IEEE Trans. Circuits and Syst. II, pp. 185-96, Mar. 2000.

[3] J. Ming and S. H. Lewis, "An 8-bit 80-Msample/s pipelined analog-todigital converter with background calibration," IEEE J. of Solid-State Circuits, pp. 1489-1497, Oct. 2001.

[4] E. J. Siragusa and I. Galton, "Gain error correction technique for pipelined analogue-to-digital converters," Electronic Letters, pp. 617618, Mar. 2000.

[5] J. P. Keane, P. J. Hurst, and S. H. Lewis, "Background interstage gain calibration technique for pipelined ADCs," IEEE Trans. Circuits and Syst. I, scheduled to appear Jan. or Feb. 2005.

[6] A. H. Sayed, Fundamentals of Adaptive Filtering. New York: John Wiley \& Sons, 2003.

[7] A. Benveniste, M. Metivier, and R. Priouret, Adaptive Algorithms and Stochastic Approximations. Berlin: Springer-Verlag, 1990. 